\title{
Saccade impairments in patients with fronto-temporal dementia
}

\author{
C Meyniel, S Rivaud-Péchoux, P Damier, B Gaymard
}

J Neurol Neurosurg Psychiatry 2005;76:1581-1584. doi: 10.1136/jnnp.2004.060392

Background: Early diagnosis of fronto-temporal dementia (FTD) is often difficult because of the non-specific presentation. Saccadic eye movements, which are mainly controlled by the frontal areas, may provide a powerful tool for the analysis of fontal lobe dysfunction. The pattern of saccadic abnormalities has not previously been investigated in patients with FTD.

Objective: To study saccade tasks in a group of 23 patients with FTD and compare the results with aged matched healthy controls.

Methods: Triggering and inhibition of reflexive prosaccades were evaluated in a prosaccade and an antisaccade task, respectively, while the ability to withhold an antisaccade during a delay was explored in a delayed antisaccade task. Patients with progressive supranuclear palsy (PSP), in whom the pattern of eye movement deficit is well documented, were studied with the same protocol. To characterise the frontal lobe dysfunction in FTD more precisely, a battery of neuropsychological tests was carried out in these patients. Results: Patients with FTD showed impaired reflexive saccade inhibition, similar to that observed in patients with PSP, and a decreased ability to withhold an antisaccade.

Conclusions: Inhibition of reflexive and voluntary saccades appears to be independently processed. A delayed antisaccade task could be useful for the early diagnosis of FTD.

$\mathrm{F}$ ronto-temporal dementia (FTD) is a neurodegenerative disorder affecting the frontal and temporal lobes. Its early diagnosis is often difficult, the initial symptoms tending to be restricted to non-specific behavioural disorders such as apathy or disinhibition. ${ }^{1-3}$ Various studies have shown that the analysis of saccadic eye movements may provide a useful tool for investigating neurological or psychiatric disorders in which the frontal lobe is impaired, as both saccade triggering and inhibition are mainly controlled by frontal cortical areas. ${ }^{4}$ Thus far, no oculomotor study has been undertaken in patients with FTD. We therefore tested various saccade paradigms in a group of patients with FTD, with special emphasis on inhibitory mechanisms. The same tasks were carried out in a group of healthy subjects and in a group of patients with progressive supranuclear palsy (PSP) in whom the pattern of saccade impairments has been well described. ${ }^{5}$

\section{METHODS}

\section{Subjects}

We recruited 23 patients with FTD meeting the LundManchester criteria ${ }^{2}$ (mean (SD) age, 67 (9) years; duration of illness, 2.9 (2.0) years), along with 14 patients with PSP meeting Litvan's criteria ${ }^{6}$ (mean age, 70 (6) years; duration of illness, 2.4 (1.6) years). We excluded patients with excessive neurological deterioration (mini-mental state examination (MMSE) score $<20$ ) or with a cerebral focal lesion assessed on magnetic resonance imaging (MRI) in the preceding two years, or both. Ten healthy subjects (mean age, 68 (9) years) with no history of neurological or psychiatric disorders served as a control group. All subjects gave their informed consent to be included in the study, which was approved by the local ethics committee.

\section{Oculomotor tests}

Eye movements were recorded by horizontal electro-oculography with our standardised protocol, as previously described. ${ }^{5}$

In the prosaccade task, subjects were asked to follow a target that jumped from a central position to an unpredictable $25^{\circ}$ right or left location. A $200 \mathrm{~ms}$ gap was interposed between central target offset and lateral target onset. This task tests the ability to trigger a reflexive saccade. Only saccade latency was measured in this task. There were 24 trials per subject.

In the antisaccade task, the same stimulus condition was used as in the prosaccade task, but subjects were instructed to trigger a saccade as soon as possible in the opposite direction to the lateral target. This task tests the ability to inhibit a reflexive prosaccade. ${ }^{7}$ Correct antisaccade latency and the percentage of (uninhibited) prosaccades were measured in this task. There were 36 trials per subject.

In the delayed antisaccade task, a variable delay (3000, 3500 , or $4000 \mathrm{~ms}$ ) was interposed between lateral target presentation and central fixation point offset. Subjects were instructed to trigger an antisaccade at the end of the delaythat is, at the extinction of the central fixation point. Successful performance of this task required the ability both to inhibit a prosaccade and to withhold an antisaccade during the delay. Consequently, a trial in which at least one saccade was triggered during the delay was scored as an error, and any such saccade was considered to be an anticipatory prosaccade or antisaccade according to its direction. We therefore determined two scores: the percentage of anticipatory prosaccade and the percentage of anticipatory antisaccade. We determined the latency of the anticipatory prosaccade and the anticipatory antisaccade with respect to target onset, and the latency of the correct antisaccade with respect to the fixation point offset. There were 24 trials per subject.

Saccade latencies were analysed by two way analysis of variance (ANOVA). Subsequent comparisons between groups were carried out with the Bonferroni correction. The percentage of responses was compared between the groups with a Mann-Whitney U test.

Abbreviations: DRS, dementia rating scale; $F A B$, frontal assessment battery; FTD, fronto-temporal dementia; MADRS, Montgomery and Asberg depression rating scale; MMSE, mini-mental state examination; PSP, progressive supranuclear palsy 


\begin{tabular}{|c|c|c|c|}
\hline Test & FTD & PSP & Controls \\
\hline \multicolumn{4}{|l|}{ Oculomotor tests } \\
\hline Latencies & $237(62)^{*}$ & $234(82)$ & $183(25)$ \\
\hline \multicolumn{4}{|l|}{ Antisaccades } \\
\hline Latencies of correct responses & $415(202)^{*}$ & $549(255)^{\star *}$ & $202(45)$ \\
\hline Per cent errors & $63(9 \text { to } 100)^{* * *}$ & $70(30 \text { to } 100)^{* * *}$ & $19(5$ to 33$)$ \\
\hline \multicolumn{4}{|l|}{ Delayed antisaccades } \\
\hline Per cent anticipatory prosaccades & $48(12 \text { to } 100)^{\star \star *}$ & $47(4 \text { to } 96)^{* *}$ & $8(0$ to 24$)$ \\
\hline Per cent anticipatory antisaccades & $34(0 \text { to } 96)^{* *} \dagger$ & $12(0$ to 33$)$ & $8(0$ to 21$)$ \\
\hline Latencies of correct responses & $802(476)^{\star *}$ & $663(310)$ & $400(155)$ \\
\hline Per cent correct responses & $75(16 \text { to } 100)^{* *}$ & $88(65$ to 100$)$ & $97(88$ to 100$)$ \\
\hline \multicolumn{4}{|l|}{ Neuropsychological tests } \\
\hline MMSE & $26.1(2.8) \dagger \dagger \dagger \S$ & $28.6(2.0)$ & - \\
\hline $\mathrm{FAB}$ & $10.5(4.3) \S$ & $12.7(2.3) \S$ & - \\
\hline MADRS & $10.6(11.3) \dagger$ & $18.5(7.1)$ & - \\
\hline Fronto-temporal behavioural scale of Lebert & $6.3(3.4) \S$ & $6.0(1.9) \S$ & - \\
\hline Mattis DRS & $120.9(16.9) \S$ & - & - \\
\hline \multicolumn{4}{|l|}{ Rolls } \\
\hline Reversal: number of reversals (RI) & $1.0(0.9) \S$ & - & - \\
\hline Reversal: last error trial (R2) & $20.1(7.9) \S$ & - & - \\
\hline Extinction (R3) & $24.0(7.2) \S$ & - & - \\
\hline \multicolumn{4}{|l|}{ Owen } \\
\hline Per cent novel sequences generated $(\mathrm{O} 1)$ & $54(42$ to 66$) \S$ & - & - \\
\hline Per cent repetitive trial after first error (O2) & $33(13$ to 54$) \S$ & - & - \\
\hline Apathy scale & $17.4(6.2) \S$ & - & - \\
\hline \multicolumn{4}{|c|}{ 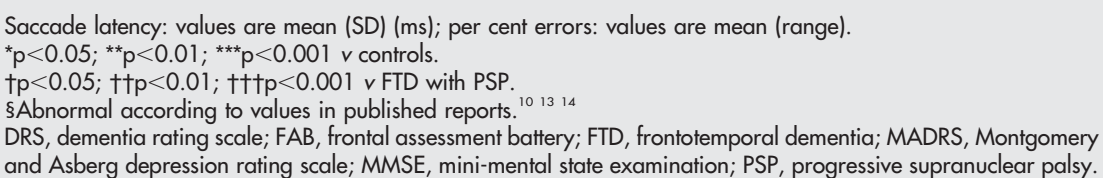 } \\
\hline
\end{tabular}

\section{Neuropsychological tests}

Cognitive functions in both FTD and PSP patients were measured by the MMSE. ${ }^{8}$ Global frontal lobe functions were assessed by a frontal assessment battery (FAB) and the fronto-temporal behavioural scale of Lebert. ${ }^{10}$ Depression was measured by the Montgomery and Asberg depression rating scale (MADRS). ${ }^{11}$ In the FTD group, global frontal lobe functions were also evaluated by the Mattis dementia rating scale (DRS). ${ }^{12}$ As the orbitofrontal, dorsolateral, and frontomedian cortices may be affected differently in patients with FTD, we also studied three additional tests in this group. In the emotion related learning test of Rolls (orbitofrontal cortex), we measured "the number of reversals completed in the first 30 trials (R1) and the trial on which the last error occurred in the first reversal (R2) and in extinction (R3)". ${ }^{13}$ In the spatial sequence generation task of Owen (dorsolateral cortex), we measured "the number of sequences generated, expressed as a percentage of 24 possible responses, and the percentage of sequences (after an error), that began with the same box used to begin the previous sequence" ${ }^{14}$ Starkstein's scale was used to quantify the level of apathy (fronto-median cortex). ${ }^{15}$ The performance of the FTD and PSP groups on neuropsychological tests was compared with a MannWhitney test. In the FTD group, the other results were analysed with respect to published reports. ${ }^{10}{ }^{13}{ }^{14}$ Correlations between neuropsychological scores and oculomotor variables were studied using the Spearman test.

\section{RESULTS}

\section{Oculomotor tasks}

Compared with the control group, saccade latency in the FTD group was increased in all three prosaccade $(\mathrm{p}<0.025)$, antisaccade $(\mathrm{p}<0.046)$, and delayed antisaccade tasks $(\mathrm{p}<0.015)$. Saccade latencies were not significantly different in the PSP and FTD groups (table 1).

In the antisaccade task, both FTD and PSP patients had an increased percentage of errors compared with control subjects (FTD, $\mathrm{p}<0.0001$; PSP, $\mathrm{p}<0.0001$ ); the results of the two patient groups were not significantly different. In the delayed antisaccade task, both PSP and FTD patients had an increased percentage of anticipatory prosaccades compared with controls (FTD, p<0.0001; PSP, p<0.004) (table l). The percentage of anticipatory antisaccades was significantly increased in the FTD group compared with both the normal group $(\mathrm{p}<0.01)$ and the PSP group $(\mathrm{p}<0.014)$, but this percentage was normal in the PSP group (table 1).

\section{Cognitive tests}

Global cognitive functions (MMSE) were more affected in the FTD than in the PSP group $(p<0.001)$. Frontal lobe functions were impaired in both FTD and PSP patients, but with no significant difference between the groups (FAB, $p<0.182$ ). The MADRS score was significantly higher in the PSP group than in the FTD group $(\mathrm{p}<0.041)$, but below the depression threshold in both groups. The results of the three regional frontal tests (Rolls, Owen, and scale of apathy) were abnormal in all FTD patients (table 1).

\section{Correlations}

Significant correlations were found between the percentage of errors in the antisaccade task and the results in the frontal lobe tests (FAB, $r=-0.75, \mathrm{p}<0.0001$; Mattis DRS, $r=-0.86$, $\mathrm{p}<0.0001$; Rolls R2, $r=0.69, \mathrm{p}<0.007$; Rolls R3, $r=0.55$, $\mathrm{p}<0.044$ ). No significant correlation was found between any of the three regional frontal tests and the percentage of anticipatory antisaccades in the delayed antisaccade task.

\section{DISCUSSION}

This is the first oculomotor study carried out on patients with FTD. Our main finding was that both FTD and PSP patients showed a decreased ability to inhibit reflexive prosaccades (antisaccade and delayed antisaccade tasks), but that only FTD patients were impaired in the ability to withhold voluntary saccades (delayed antisaccade task, fig 1A). 


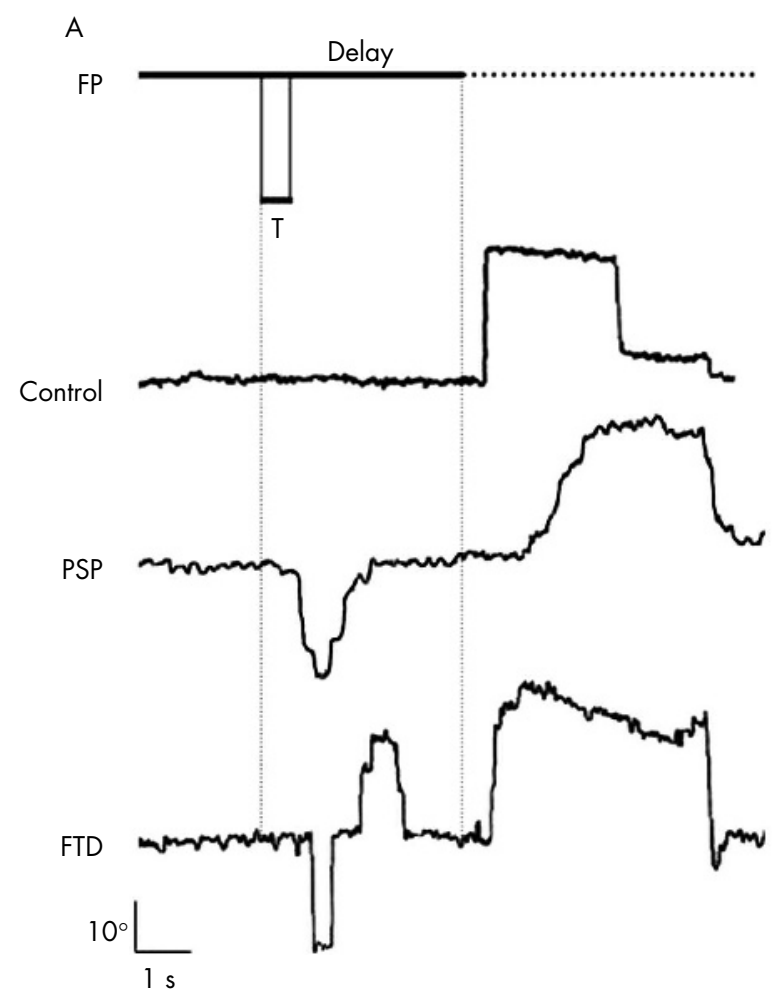

schizophrenic or attention deficit/hyperactivity disorder. ${ }^{4}$ The high percentage of uninhibited prosaccades observed in FTD patients in both antisaccade and delayed antisaccade tasks thus suggests that the dorsolateral prefrontal cortex is affected in this disease. The correlation between the percentage of errors in the antisaccade task and the global frontal tasks (FAB, Mattis DRS) is consistent with an involvement of the dorsolateral prefrontal cortex in all these tasks. The correlation found between the percentage of errors in the antisaccade task and the Rolls test was unexpected. It could reflect the influence of motivation in the successful performance of the antisaccade task.

In both FTD and PSP patients, anticipatory prosaccades in the delayed antisaccade task occurred shortly after target presentation (fig $1 \mathrm{~B}$ ). It might therefore be proposed that dorsolateral prefrontal cortex inhibitory functions are mainly exerted on reflexive, externally triggered movements-that is, during a short time window following target presentation. However, PSP patients were able to withhold voluntary saccades in the delayed antisaccade task (fig 1B). As the dorsolateral prefrontal cortex is the frontal area mainly affected in PSP, it does not seem to be essential for the suppression of non-reflexive internally triggered movements. ${ }^{5}$ An opposite pattern has been reported in patients with Gilles de la Tourette syndrome-that is, normal reflexive prosaccade inhibition but impaired ability to withhold voluntary saccades. ${ }^{16}$ These results are consistent with the hypothesis that inhibition of externally triggered movements and inhibition of internally triggered movements are processed independently. Hence, the markedly increased percentage of anticipatory antisaccades in FTD patients (delayed antisaccade task) probably resulted from a dysfunction lying outside the dorsolateral prefrontal cortex. The results of the neuropsychological tests indicate that frontal lobe dysfunction in our FTD patients was not restricted to the dorsolateral prefrontal cortex but extended to the orbitofrontal and fronto-median cortices as well. Inability to withhold voluntary saccades could therefore result from a dysfunction of these areas, individually or in association. Unfortunately, we did not find any correlation between the three regional frontal lobe tests and the percentage of anticipatory antisaccades. This negative result may be explained by an excessive sensitivity of these cognitive tests. Further investigation of this paradigm in patients with focal frontal lesions is therefore needed to clarify this issue.

In conclusion, our study suggests that oculomotor paradigms may be of interest for the study of FTD patients provided that this abnormality is present at an early stage of the disease.

\section{ACKNOWLEDGEMENTS}

We thank Dr Lucette Lacomblez, Dr Marie Sarazin, Dr Bruno Dubois, and Matthias Pessiglione for their helpful cooperation in this study.

\section{Authors' affiliations}

C Meyniel, P Damier, Service de Neurologie, CHU de Nantes, Nantes, France

S Rivaud-Péchoux, INSERM U679, Hôpital de la Salpêtrière, Paris,

Figure 1 Delayed antisaccade task. (A) Typical eye movement recordings. The upper trace represents the paradigm. (B) Distribution of anticipatory prosaccade (PS) and anticipatory antisaccade (AS) latencies in the FTD and PSP groups. FP, fixation point; FTD, fronto-temporal dementia; PSP, progressive supranuclear palsy; T, target.

Reflexive prosaccade inhibition is abnormal in a broad spectrum of diseases in which the dorsolateral prefrontal cortex is damaged. Poor antisaccade performance is thus observed in patients with focal frontal lobe lesions, in neurodegenerative diseases such as PSP, Huntington's disease, and Alzheimer's disease, and in patients with

B Gaymard, Service d'Explorations Fonctionnelles du Système Nerveux, Hôpital de la Salpêtrière

Competing interests: none declared

Correspondence to: Dr B Gaymard, INSERM U679 (formerly U289), Hôpital de la Salpêtrière, 75651 Paris Cedex 13, France; gaymard@ccr.jussieu.fr

Received 6 December 2004

In revised form 24 February 2005

Accepted 24 February 2005 


\section{REFERENCES}

1 McKhann GM, Albert MS, Grossman M, et al. Clinical and pathological diagnosis of frontotemporal dementia: report of the Work Group on Frontotemporal Dementia and Pick's Disease. Arch Neurol 2001;58:1803-9.

2 Miller BL, Ikonte C, Ponton M, et al. A study of the Lund-Manchester research criteria for frontotemporal dementia: clinical and single-photon emission CT correlations. Neurology 1997;48:937-42.

3 Rosso SM, Donker Kaat L, Baks T, et al. Frontotemporal dementia in The Netherlands: patient characteristics and prevalence estimates from a population-based study. Brain 2003;126:2016-22.

4 Everling S, Fischer B. The antisaccade: a review of basic research and clinical studies. Neuropsychologia 1998;36:885-99.

5 Pierrot-Deseilligny C, Rivaud S, Pillon B, et al. Lateral visually-guided saccades in progressive supranuclear palsy. Brain 1989:112:471-87.

6 Litvan I, Agid Y, Calne D, et al. Clinical research criteria for the diagnosis of progressive supranuclear palsy (Steele-Richardson-Olszewski syndrome): report of the NINDS-SPSP international workshop. Neurology 1996;47:1-9.

7 Pierrot-Deseilligny C, Muri RM, Ploner CJ, et al. Decisional role of the dorsolateral prefrontal cortex in ocular motor behaviour. Brain 2003; 126:1460-73.
8 Folstein MF, Folstein SE, McHugh PR. "Mini-mental state". A practical method for grading the cognitive state of patients for the clinician. J Psychiatr Res 1975; 12:189-98.

9 Dubois B, Slachevsky A, Litvan I, et al. The FAB: a frontal assessment battery at bedside. Neurology 2000:55:1621-6.

10 Lebert $F$, Pasquier F, Souliez L, et al. Frontotemporal behavioral scale. Alzheimer Dis Assoc Disord, 1998; 12:335-9.

11 Montgomery SA, Asberg M. A new depression scale designed to be sensitive to changes. Br J Psychiatry 1979;134:382-9.

12 Mattis S. Dementia rating scale. Professional manual. Florida: Psychological Assessment Resources, 1988.

13 Rolls ET, Hornak J, Wade D, et al. Emotion-related learning in patients with social and emotional changes associated with frontal lobe damage. J Neurol Neurosurg Psychiatry 1994;57:1518-24.

14 Owen A, Hodges J, Summers B, et al. Dopamine-dependent frontostriatal planning deficits in early Parkinson's disease. Neurology 1995;9:126-40.

15 Starkstein SE, Mayberg HS, Preziosi TJ, et al. Reliability, validity, and clinical correlates of apathy in Parkinson's disease. J Neuropsychiatry Clin Neurosci 1992;4:134-9.

16 LeVasseur AL, Flanagan JR, Riopelle RJ, et al. Control of volitional and reflexive saccades in Tourette's syndrome. Brain 2001;124:2045-58.

\section{NEUROLOGICAL PICTURE}

\section{Spinal anterior artery territory infarction simulating an acute myocardial infarction}

$\Lambda$ 59 year old man with history of smoking, hypertension, diabetes mellitus, and hypercholesterolemia presented suddenly with numbness and weakness of both upper limbs. A few minutes after that he felt an intense and oppressive chest pain accompanied by profuse diaphoresis, severe hypotension, bradycardia, and low level of consciousness. No elevated damage cardiac markers or electrocardiographic changes were registered. Aortography ruled out an aortic dissection whereas coronary angiography showed a moderate stenosis in the left coronary trunk, which was stented.

An adequate neurological examination could be performed only after 48 hours, when the patient was extubated. He had a bilateral, predominantly proximal (MRC grade $2 / 5$ versus 4 / 5 for the distal muscles), flaccid arm paresis, with absent reflexes. Muscular balance and deep tendon reflexes in both lower limbs were normal. All sensory modalities were preserved and there were neither autonomic abnormality nor Babinski sign.

Cranial MRI ruled out bilateral brain infarctions in the border zone between middle and anterior cerebral artery territories - the most frequent aetiology of acute man-in-thebarrel syndrome. Surprisingly, cervical MR demonstrated a spinal anterior artery territory infarction between C2 and C7 levels involving exclusively both anterior horns of the spinal cord (panels A and B), whose cells are known to have a special vulnerability to ischaemia. ${ }^{1-3}$ Magnetic angioresonance made evident a severe atheromatosis within the right vertebral artery (panel C).

Prominent vegetative symptoms and chest pain at the onset provoked an initial misdiagnosis of acute myocardial infarction despite the absence of compatible biochemical and electrocardiographic changes, but they can be plausibly explained by a transient ischaemia of intermediate-lateral horns and of spinothalamic tracts at the lower segments of cervical cord, respectively. ${ }^{4}$

Cervical cord infarction should be considered as a cause of acute bilateral arm paresis and included in the differential diagnosis of chest pain with accompanying neurological symptoms together with myocardial infarction and aortic dissection
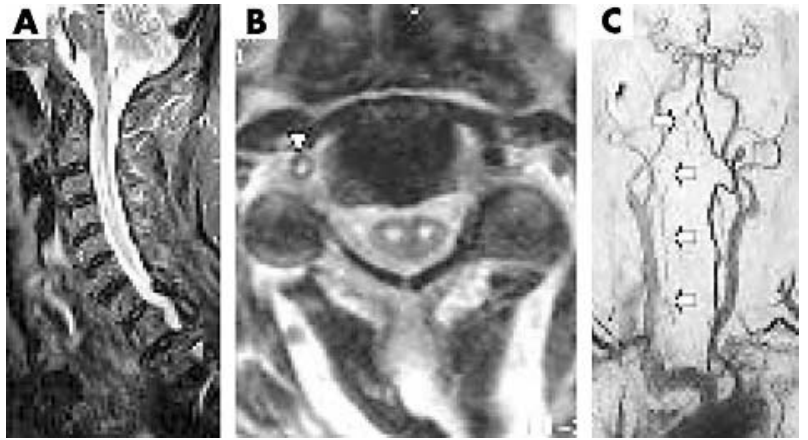

(A) MRI T2-weighted sagittal section: hyperintense pencil-like lesion in anterior portion of spinal cord from C2 to C7 level. (B) MRI T2-weighted axial section: bilateral hyperintense signal in anterior horns (snake-eyes image). Note also a high intensity signal in right vertebral hole (arrow head). (C) Magnetic angioresonance: severe atheromatosis in right vertebral artery (arrows)

A Serrano-Pozo, G Sanz-Fernández, E Martínez-Fernández Department of Neurology, University Hospital Virgen del Rocío, Seville (Spain) J Nevado-Portero Department of Cardiology, University Hospital Virgen del Rocío, Seville (Spain)

Correspondence to: Alberto Serrano Pozo, Plaza Ruiz de Alda n 7 10D, 41004 Seville (Spain); albserrap@yahoo.es

Competing interests: none declared

\section{References}

1 Pullicino P. Bilateral distal upper limb amyotrophy and watershed infarcts from vertebral dissection. Stroke 1994;25:1870-2.

2 Berg D, Müllges W, Koltzenburg M, et al. Man-in-the-barrel syndrome caused by cervical spinal cord infarction. Acta Neurol Scand 1998;97:417-9.

3 Stapf C, Mohr JP, Straschill M, et al. Acute bilateral arm paresis. Cerebrovasc Dis 2000; 10:239-43.

4 Cheshire WP Jr. Spinal cord infarction mimicking angina pectoris. Mayo Clin Proc 2000;75:1197-9.

5 Combarros O, Vadillo A, Gutiérrez-Pérez R, et al. Cervical spinal cord infarction simulating myocardial infarction. Eur Neurol 2002;47:185-6. 


\section{PostScript}

\section{LETTERS}

\section{Paraneoplastic cerebellar degeneration in olfactory neuroepithelioma}

Anti-Hu antibody was first discovered in patients with paraneoplastic encephalomyelitis associated with small cell lung cancer (SCLC). This antibody recognises proteins comprised in the $\mathrm{Hu}$ family expressed by neuronal cells as well as SCLC. After the first report, anti-Hu antibody was found in other neoplasms including prostate and breast cancer, adrenal carcinoma, chondromyxosarcoma, neuroblastoma, and neuroendocrine neoplasms at other sites. ${ }^{1}$ Olfactory neuroepithelioma $(9523 / 3)^{2}$ is thought to differ from classic neuroblastoma $(9500 / 3)$ in its expression pattern of tyrosine hydroxylase, MYCN amplification, and fusion of the Ewing sarcoma gene and the Friend leukaemia virus integration 1 gene or the ETS related gene.

Anti-Hu antibody in association with olfactory neuroepithelioma has not been reported previously. We report a patient with cerebellar ataxia that paralleled the recurrence of the tumour. Serum and cerebrospinal fluid (CSF) from the patient contained anti-Hu antibody, and the olfactory neuroepithelioma resected from the patient expressed $\mathrm{Hu}$ antigen.

\section{CASE REPORT}

Seven years before admission, a 65 year old man presented with olfactory neuroepithelioma that had invaded the orbit and frontal lobe. The tumour was dissected surgically, and dura mater graft was not used in the surgery. The patient underwent irradiation (total dose of $50 \mathrm{~Gy}$ ). The tumour recurred at the parotid gland in January 2001, and there was gait instability. The patient consulted a neurologist, but there was no specific finding. The recurrent tumour was surgically dissected; however, the instability progressed rapidly, and at the patient's admission in November 2001, he needed support when walking. There was neither alcoholism nor family history of cerebellar ataxia. His parents were not consanguineous.

General physical examination was negative. There was no lymphoadenopathy. He was alert and mentally normal. Olfactory sensation had been decreased since the first surgery, there was a downbeat nystagmus, and muscle strength was maximum. Both superficial and deep sensation were normal. Deep tendon reflex was symmetrical and normal, Romberg test was negative, and no pathological reflex was found. Nose-fingernose test was normal, but heel-shin test was poor. Dysmetria was marked in both legs. His gait was wide based and ataxic, and tandem gait was impossible. There was no dysarthria.

Haematological studies, blood chemical analyses, and serological studies were normal. Tumour markers including $\alpha$-fetoprotein, prostate specific antigen, pro-gastrin releasing peptide, neurone specific enolase, sialyl Lewis (a) (CA19-9), and sialyl Lewis (x) (SLX) were within normal limits. Levels of vitamin Bl and B12 were normal. Protein level in cerebrospinal fluid (CSF) was increased to $105 \mathrm{mg} / \mathrm{dl}$ with normal cellularity. Myelin basic protein and oligoclonal IgG band was negative. IgG index was 0.6. No malignant cells were found in the CSF. Nerve conduction study was normal. Short sensory evoked potentials of upper and lower limbs were normal. Electroencephalogram showed beta rhythm at the bilateral frontal region, with otherwise normal findings.

Computed tomography (CT) showed no lung tumour. Magnetic resonance imaging (MRI) showed bilateral leukoaraiosis at bilateral frontal lobes that had been present since after the first surgery. The cerebellum was slightly atrophic.

Titres of anti-Hu antibody in the serum and CSF were 1:1920 and 1:64, respectively (indirect immunofluorescence and Western blotting for recombinant $\mathrm{HuD}$ ). Serum:CSF antibody titre ratio was 30 . The ratio for (CSF/serum antibody titre)/(CSF/serum albu$\min )$ was 1.8 . These values indicated that intrathecal synthesis of anti-Hu antibody had stopped at this time point. Other antineuronal antibodies including anti-Yo, Ri, CV2, Tr, Ma, amphiphysin, and glutamic acid decarboxylase were all negative. Systemic examination including ${ }^{67} \mathrm{Ga}$-citrate scintigraphy did not disclose malignant tumours. Immunohistochemistry with anti-HuD antibody (Santa Cruz, sc-5977, ×100) revealed that a part of the tumour expressed $\mathrm{Hu}$ protein (fig 1).

Over the course of 4 years after discharge, the cerebellar ataxia did not worsen further in the absence of immunological treatment. Follow up thoracic CT and tumour marker study did not disclose other malignant tumours. There was no evidence of the recurrence of olfactory neuroepithelioma.

\section{CONCLUSION}

This patient presented cerebellar ataxia of the trunk and lower limbs that progressed rapidly within approximately 6 months after the second surgery and stabilised thereafter.

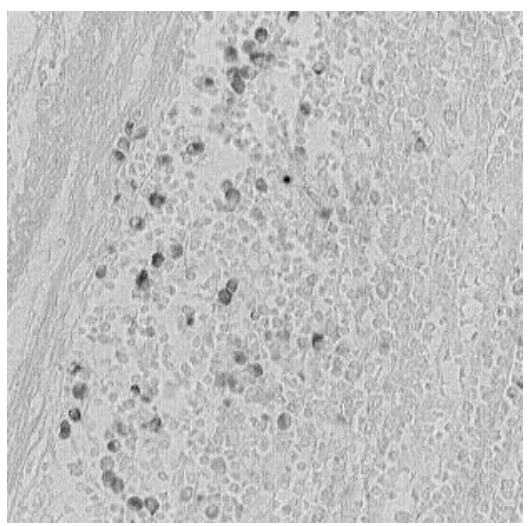

Figure 1 Immunohistochemistry using antiHuD antibody. A part of the patient's tumour expressed HuD antigen $(\times 400)$.
This clinical course is not inconsistent with the natural course of paraneoplastic cerebellar degeneration. Although isolated cerebellar ataxia in anti-Hu antibody positive patients is rare (4/200 patients), ${ }^{1}$ a high titre of serum anti-Hu antibody (1:1920) corroborated the diagnosis of paraneoplastic syndrome. ${ }^{1}$ The expression of the HuD protein by the olfactory neuroepithelioma confirmed the diagnosis.

Olfactory neuroepithelioma is a neuroectodermal neoplasm that arises from the olfactory epithelium. It is distinguished from classic neuroblastoma as described by Sorensen et al. ${ }^{3}$ Unlike neuroblastoma, olfactory neuroepithelioma shows differentiation to the neural processes and glandular structure and is rarely associated with catecholamine secretion. In addition, olfactory neuroepithelioma expresses epithelial markers such as cytokeratin and a $34 \mathrm{kDa}$ epithelial membrane glycoprotein recognised by monoclonal antibody named Ber-EP4. The tumour in this case expressed both Ber-EP4 and cytokeratin (see Okabe et al ${ }^{4}$, case no. 6). Moreover, it also expressed luteinising hormone releasing hormone. The expression pattern of Ber-EP4 and cytokeratin was heterogeneous in this tumour. ${ }^{4}$ These findings suggest that the tumour in this case had arisen from the olfactory placode and was distinct from classic neuroblastoma arising from the neural crest. ${ }^{5}$ This neuroepithelial tumour has not been reported to be associated with paraneoplastic syndrome. Our data clearly demonstrate the expression of $\mathrm{Hu}$ antigen by the olfactory neuroepithelioma cells and the presence of $\mathrm{Hu}$ antibody in his serum and CSF. It is interesting that neurological manifestations developed in parallel with the recurrence of the tumour. The recurrence might have enhanced immune response. Despite resection of the recurrent tumour, the cerebellar ataxia worsened for several months after surgery. However, it did not progress thereafter. In patients with neurological symptoms and $\mathrm{Hu}$ antibody, olfactory neuroepithelioma should be considered when a neoplasm is not found at the common sites such as the lung or breast.

K Maeda, T Sasaki, Y Murata, M Kanasaki, T Terashima, H Kawai, H Yasuda

Division of Neurology, Department of Medicine, Shiga University of Medical Science, Otsu, Shiga, Japan 520-2192

H Okabe

Department of Clinical Laboratory Medicine, Shiga University of Medical Science, Otsu, Shiga, Japan

520-2192

K Tanaka

Department of Neurology, Brain Research Institute, Niigata University, Niigata, Japan

Correspondence to: Kengo Maeda, Division of Neurology, Department of Medicine, Shiga University of Medical Science, Ołsu, Shiga, Japan 520-2192; kengo@belle.shiga-med.ac.jp

doi: 10.1136/jnnp.2005.066977

Competing interests: none 


\section{REFERENCES}

1 Graus F, Keime-Guibert F, Reñe R, et al. Anti-Huassociated paraneoplastic encephalomyelitis: analysis of 200 patients. Brain 2001; 124:1138-48

2 In: Kleihues P, Cavenee WK, eds. World Health Organisation classification of tumours: pathology and genetics of tumours of the nervous system. Lyon: IARC Press, 2000

3 Sorensen PHB, Wu JK, Berean KW, et al. Olfactory neuroblastoma is a peripheral primitive neuroectodermal tumor related to Ewing sarcoma. Proc Natl Acad Sci USA 1996;93:1038-43.

4 Okabe H, Okubo T, Ochi Y. On the origin and diagnostic criteria of olfactory neuroblastoma. Acta Histochem Cytochem 1997;30:181-8.

5 Okabe H, Okubo T, Ochi Y. Expression of epithelial membrane glycoprotein by neurons arising from olfactory plate through development. Neuroscience 1996;72:579-84.

\section{The collagen $1 \mathrm{~A} 2$ polymorphism rs42524, which is associated with intracranial aneurysms, shows no association with spontaneous cervical artery dissection (sCAD)}

In the last decade, extracranial spontaneous cervical artery dissection (SCAD) became increasingly recognised as a common cause of juvenile stroke. Hereditary connective tissue diseases such as Ehlers-Danlos syndrome type IV (EDS IV) and Marfan syndrome can be associated with SCAD and clinical signs of mild connective tissue weakness have been described in some patients with sCAD. ${ }^{1}$ Brandt and co-workers found connective tissue aberrations mainly affecting the collagen fibres in skin biopsies of approximately $60 \%$ of patients with sCAD. ${ }^{2}$ Similar skin aberrations were found in patients with intracranial aneurysms (IA). ${ }^{3}$ A familial association of IA and SCAD has been observed in a few families. ${ }^{4}$ These findings suggest that connective tissue abnormalities are common to both diseases and might predispose to IA as well as to sCAD. Recently, association between the functional coding single nucleotide polymorphism (SNP) rs42524 in the collagen lA2 (COLlA2) gene and IA has been described. ${ }^{5}$ The SNP rs42524 causes a base change Gl645C and an amino acid change alanine 549 to proline in the COLlA2 gene (cDNA NM_000089).

In this study we test the hypothesis that the SNP rs42524 is also associated with SCAD. We studied 144 unrelated patients with extracranial sCAD (86 men, mean age 47.4 (standard deviation (SD) 11.5 ) years; 89 SCAD of the internal carotid artery (ICA), 49 of the vertebral artery (VA), and 6 of the ICA and the VA; patients without known specification of the dissected artery were excluded) and 162 healthy age and sex matched blood donors (98 men, mean age 43.5 (SD 8.7) years) from the province of North Rhine-Westfalia, Germany. All patients were enrolled retrospectively by the Department of Neurology of the University of Münster (described in Konrad et $a l^{6}$ ) and the Department of Neurology of the Alfried Krupp Hospital, Essen in essentially the same way. The diagnosis of SCAD required clinical symptoms suggestive of SCAD and either evidence of intramural haematoma on MRI examination of the cervical arteries with

Table 1 COL1A2 rs42524 genotypes

\begin{tabular}{|c|c|c|c|c|c|c|c|}
\hline \multirow[b]{2}{*}{ Group } & \multicolumn{4}{|c|}{ Genotypes } & \multicolumn{3}{|l|}{ Alleles } \\
\hline & GG & CG & $\mathrm{CC}$ & $p$ & G & $\mathrm{C}$ & $p$ \\
\hline All sCAD & $87(61)$ & $48(33)$ & $9(6)$ & 0.99 & $222(77)$ & $66(23)$ & 1.00 \\
\hline$s C A D$ of ICA & $57(64)$ & $28(31)$ & $4(5)$ & 0.79 & $142(80)$ & $36(20)$ & 0.57 \\
\hline sCAD of VA & $27(55)$ & $17(35)$ & $5(10)$ & 0.59 & 71 (72) & $27(28)$ & 0.41 \\
\hline Controls & $98(61)$ & $54(33)$ & $10(6)$ & - & 250 (77) & $74(23)$ & - \\
\hline
\end{tabular}

Values are $\mathrm{n}(\%)$, except for the $\mathrm{p}$ values. ICA, internal carotid artery; VA, vertebral artery. Six individuals suffered from concomitant SCAD of ICA and VA and were excluded from the subgroup analysis.

transverse sections through the neck or typical signs of SCAD on intra-arterial digital subtraction angiography. Subjects gave informed consent and the study was approved by the local ethics committee. Genomic DNA was extracted from peripheral blood lymphocytes. Genotyping was performed using a PCR-RFLP assay. Genotype and allele differences between groups were assessed using $\chi^{2}$ statistics. Hardy-Weinberg equilibrium was calculated using an online resource (http://kursus.kvl.dk/shares/vetgen). Probability (p) values of less than 0.05 were considered statistically significant. The study had $84 \%$ power to detect an association with an OR of 3.19 described in familial Japanese IA and $58 \%$ to detect an association with an OR of 1.99 described in sporadic Japanese IA with 95\% confidence (PS program; http:// statgen.iop.kcl.ac.uk/gpc/). The genotype and allele frequencies of the SNP rs42524 are shown in table 1. Genotype frequencies in the patients, as well as in the control group, were in good agreement with Hardy-Weinberg equilibrium $(p=0.49$ for controls and $\mathrm{p}=0.47$ for patients). Neither genotype nor allele frequencies showed significant differences between SCAD patients and controls in the whole sample, after stratification for the affected vessel (ICA or VA) (table 1) or after stratification for gender (results not shown).

\section{Comment}

The COLlA2 gene is located on chromosome 7q22.1, a chromosomal region showing linkage with IA. ${ }^{7}$ The SNP rs42524 in the COLlA2 gene showed strong association with IA in a Japanese IA cohort and supposedly influences the thermal stability of collagen. ${ }^{5}$ We investigated this SNP in SCAD patients because of the presumed pathophysiological similarities between both diseases. We did not find an association between SCAD and the SNP rs42524. However, it can not be excluded that this is due to ethnically determined differences in allele frequencies, because the chromosome $7 \mathrm{q}$ locus for IA as well as the association with the SNP rs42524 were originally found in a Japanese sample in which the $\mathrm{C}$ allele has a frequency of only $2.7 \%$ in the control group, while we studied a Caucasian population in which the $\mathrm{C}$ allele had a frequency of $23 \% .^{5}$ The sample size of the Japanese study (260 IA patients) and of our own study (144 SCAD) were of the same order of magnitude. In summary, this study renders it unlikely that the SNP rs42524 plays a major role in the pathogenesis of SCAD in Caucasians. However, this study does not exclude the possibility that other polymorphisms in the COLlA2 gene, which is a very large gene covering $37000 \mathrm{bp}$ of genomic DNA, are associated with SCAD.
G Kuhlenbäumer

Leibniz-Institute of Atherosclerosis Research, University of Münster, Münster, Germany

C Konrad Department of Psychiatry, University of Münster, Münster, Germany

S Krämer Department of Radiology, University of Münster, Münster, Germany

B Kis

Department of Neurology, Alfried Krupp Hospital, Essen, Germany

D Nabavi, R Dittrich

Department of Neurology, University of Münster, Münster, Germany

E B Ringelstein

Leibniz-Institute of Atherosclerosis Research,

University of Münster, Münster, Germany

Correspondence to: $\operatorname{Dr}$ G Kuhlenbäumer, LeibnizInstitute of Atherosclerosis Research, University of Münster, Domagkstr. 3, D-48149 Münster, Germany; gkuhlen@uni-muenster.de

doi: 10.1136/jnnp.2005.065847

The research reported in this manuscript has partly been sponsored by the Bundesministerium für Bildung und Forschung (BMBF) within the stroke competence network

Competing interests: none declared

\section{Electronic-database information}

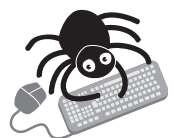

The following websites have been mentioned in this letter: http://kursus.kvl.dk/shares/ vetgen and $h+t p: / /$ statgen.iop.kcl.ac.uk/gpc/.

\section{References}

1 Schievink WI, Wijdicks EF, Michels WV, et al. Heritable connective tissue disorders in cervical artery dissections: a prospective study. Neurology 1998;50:1166-9.

2 Brandt T, Hausser I, Orberk E, et al. Ultrastructural connective tissue abnormalities in patients with spontaneous cervicocerebral artery dissections. Ann Neurol 1998;44:281-5

3 Grond-Ginsbach C, Schnippering H, Hausser I, ef al. Ultrastructural connective tissue aberrations in patients with intracranial aneurysms. Stroke 2002;33:2192-6.

4 Schievink WI, Mokri B, Michels VV, et al. Familial association of intracranial aneurysms and cervical artery dissections. Stroke 1991;22:1426-30.

5 Yoneyama T, Kasuya H, Onda H, et al. Collagen type l alpha2 (COL1A2) is the susceptible gene for intracranial aneurysms. Stroke 2004;35:443-8.

6 Konrad C, Langer C, Muller GA, et al. Protease inhibitors in spontaneous cervical artery dissections. Stroke 2005;36:9-13. 
7 Onda H, Kasuya H, Yoneyama T, et al. Genomewide-linkage and haplotype-association studies map intracranial aneurysm to chromosome 7q1 1. Am J Hum Genet $2001 ; 69: 804-19$.

\section{BOOK REVIEWS}

\section{Essential Neurosurgery, 4th edition}

Edited by Andrew H Kaye. Published by Oxford University Press, Oxford2005, £29.95 (softcover), pp 297. ISBN 1405116412

This is a short running textbook running to 280 pages of narrative on the basic principles of neurosurgery written by a Professor of neurosurgery in Melbourne. The author indicates that the book describes his own practice, which he describes as the best from the differing North American and European approaches, incorporating some of the unique advances and philosophies of the Asia Pacific Rim region.

It is a nicely laid out and well written book It covers all the basic aspects of neurosurgery and someone who reads it from cover to cover will have a good basic grounding in the speciality. I would recommend it to anyone just about to take up an SHO post in neurosurgery and would also advise career neurologists to read it early in their career, to give them a sense of what their neurosurgical colleagues will be up to. Keen medical students who wish to inform themselves well about neurosurgery would also benefit from reading it, although, given the short time that students are exposed to neurosciences nowadays, they may consider it too detailed for the basic level of knowledge they are expected to acquire. I think neurosurgical registrars should already be familiar with the concepts outlined in the book before they start their training.

I suppose it is a sad reflection on our times that I attended a meeting recently with a medical negligence lawyer who was carrying a copy of the second edition of Professor Kaye's book. This is another category of potential purchasers, to whom the third edition can be recommended.

P Richards

\section{The neurology of AIDS, second edition}

Edited by Howard E Gendelman, Igor Grant, Ian Paul Everall, Stuart A Lipton, Susan Swindells. Published by Oxford University Press, Oxford, 2005, £145.00 (hardcover), pp 829. ISBN 0-19-852610-5

Today nearly 40 million people are infected with HIV, 95\% living in the developing world. Since the first edition of The Neurology of AIDS in 1999 there has been considerable progress in understanding HIV. However despite rapid advances in highly active anti-retroviral therapy (HAART) neurological disease continues often in a more chronic form. The recent major advances in basic and clinical research are reflected in the extensive new information presented in the second edition of this comprehensive book.

The previous four sections have now been expanded to 12 sections divided into several chapters with multiple contributors, including clinicians, patients, and healthcare professionals

The book starts with a panel discussion setting the scene for the subsequent text and emphasising the challenges for the future.

The first five sections cover advances in basic research on HIV including molecular and cellular biology, immunology, vaccines, the blood brain barrier, and animal models and mechanisms of neurotoxicity including host factors and cellular factors. Parallels are drawn with other neurodegenerative diseases. Section 6 is a poignant collection of patients' accounts of living with neurological complications of AIDS. The next section deals comprehensively with the clinical aspects including dementia, more subtle cognitive disorders seen since the introduction of HAART, spinal cord disease, peripheral neuropathy, myopathy, neoplasms, opportunistic infection, and psychiatric disorders. A section on diagnostics follows including imaging and CSF markers. There is then an expanded paediatric section followed by discussion of antiretroviral and adjunctive drug treatment. The last section reviews the social and behavioural consequences of HIV infection including legal and ethical issues.

The book is very well illustrated throughout.

This outstanding book therefore provides an up to date comprehensive review of the basic science and the clinical aspects of the neurology of AIDS whilst emphasising future likely developments.

It is highly recommended for clinicians, research scientists, students, and other professionals involved in the care of these patients.

J Ball

\section{Classification and diagnosis of headache disorders}

Edited by Jes Olsen. Published by Oxford University Press, Oxford, 2005, £85.00 (hardcover), pp 311. ISBN 0-19-856590-9

This volume of Frontier's in Headache Research focuses on the application in research and clinical practice of the International Classification of Headache Disorders II (2004).

Section I presents epidemiological considerations, general principles in headache classification, and use of tools such as questionnaires, structured interviews, diaries, and diagnostic software programmes. Section II presents the sub-classification of migraines with and without aura and chronic migraine, probably the most well supported by epidemiological and genetic evidence.

In section III fervent debate continues about the entity "Tension-type headache" and nuances in classification-infrequent, frequent, and chronic tension-type headache. The diagnostic and pathophysiological basis for the core trigeminal autonomic cephalalgias (cluster headache, paroxysmal hemicrania, and SUNCT) is followed by syndromes that occupy the fringe of accepted sub-classifications-for example, hypnic headache, haemicrania continua, and new persistent daily headache. There is elaboration on a more pragmatic and intuitive ordering of other primary headache disorders such as idiopathic stabbing, cough, exertional, and sexual headache.

Sections IV and V provide the most interesting and thought provoking aspect of headache classification - the secondary headaches-undoubtedly the least evidence-based and consequently the most wanting in reclassification. The chapters discuss the literature and clinical characteristics of disorders such as post-traumatic headache, headache associated with substance (medication) use, infection, and vascular disorders. The chapter "Cranial Neuralgias and Central Causes of Pain" is superbly written. It dispels the myths of ophthalmoplegic "migraine", Eagle's syndrome, and Vidian neuralgia (was it all cluster headache?), while clarifying the phenotype of neuralgia associated with single nerves and their branches-for example, supraorbital neuralgia.

Section VI elaborates upon the successes and difficulties of implementation of ICDH II in practice and research. As one author points out, if a classification is not user friendly, it won't be used. Therefore, the call for a revised shortened classification for clinical practice, reserving the complexities of diagnostic hierarchy to the researcher, is required.

The preface concludes with, "Hopefully it (the current volume) will be useful and interesting reading for all those caring for headache patients as well as for researchers in headache, and others with a general interest in disease classification". It adeptly delivers as intended. It is easy to read, informative, provides helpful tools for the practising clinician, and provides further insights into the developing field of headache research.

A Bahra

\section{CORRECTIONS}

doi: 10.1136/jnnp.2004.062455corrl

A Serrano-Pozo, J Nevado-Portero, G SanzFernández, et al. Spinal anterior artery territory infarction simulating an acute myocardial infarction ( $J$ Neurol Neurosurg Psychiatry 2005;76:1584). The authors of this Neurological Picture were mistakenly grouped according to their affiliation. The correct ordering of the authors is: A SerranoPozo, J Nevado-Portero, G Sanz-Fernández, E Martínez-Fernández.

\section{doi: 10.1136/jnnp.2004.059212corr l}

A Ragoschke-Schumm, H Axer, C Fitzek, et al. Intracerebral haemorrhage in CADASIL (J Neurol Neurosurg Psychiatry 2005;76: 1606-7). The authors of this Letter were mistakenly grouped according to their affiliation. The correct ordering of the authors is: A Ragoschke-Schumm, H Axer, C Fitzek, M Dichgans, N Peters, J Mueller-Hoecker, $\mathrm{O} \mathrm{W}$ Witte, S Isenmann. 